\title{
COEFFICIENT ESTIMATES FOR BAZILEVIČ FUNCTIONS OF BI-PRESTARLIKE FUNCTIONS
}

\author{
ABBAS KAREEM WANAS
}

Received 22 December, 2019

\begin{abstract}
In the present article, we introduce and study two certain classes of holomorphic prestarlike and bi-univalent functions associated with Bazilevič function. We determinate upper bounds for the Taylor-Maclaurin coefficients $\left|a_{2}\right|$ and $\left|a_{3}\right|$ for functions belonging to these classes. Further we point out certain special cases for our results.
\end{abstract}

2010 Mathematics Subject Classification: 30C45; 30C50

Keywords: holomorphic function, bi-univalent function, coefficient estimates, prestarlike function, Bazilevič function

\section{INTRODUCTION}

Let $\mathcal{A}$ indicate the collection of all holomorphic functions in the unit disc $U=\{z \in \mathbb{C}:|z|<1\}$ that have the form:

$$
f(z)=z+\sum_{k=2}^{\infty} a_{k} z^{k}
$$

We also denote by $S$ the sub-collection of the set $\mathcal{A}$ containing of functions in $U$ satisfying (1.1) which are univalent in $U$.

A function $f \in \mathcal{A}$ is called starlike of order $\delta(0 \leq \delta<1)$, if

$$
\operatorname{Re}\left\{\frac{z f^{\prime}(z)}{f(z)}\right\}>\delta, \quad(z \in U) .
$$

For $f \in \mathcal{A}$ given by (1.1) and $g \in \mathcal{A}$ defined by

$$
g(z)=z+\sum_{k=2}^{\infty} b_{k} z^{k}
$$

the "Hadamard product" of $f$ and $g$ is defined (as usual) by

$$
(f * g)(z)=z+\sum_{k=2}^{\infty} a_{k} b_{k} z^{k}, \quad(z \in U) .
$$

(C) 2020 Miskolc University Press 
Ruscheweyh [8] defined and considered the family of "prestarlike functions" of order $\delta$, which are the function $f$ such that $f * I_{\delta}$ is a starlike function of order $\delta$, where

$$
I_{\delta}(z)=\frac{z}{(1-z)^{2(1-\delta)}}, \quad(0 \leq \delta<1, z \in U) .
$$

The function $I_{\delta}$ can be written in the form:

$$
I_{\delta}(z)=z+\sum_{k=2}^{\infty} \varphi_{k}(\delta) z^{k}
$$

where

$$
\varphi_{k}(\delta)=\frac{\prod_{i=2}^{k}(i-2 \delta)}{(k-1) !}, \quad k \geq 2 .
$$

We note that $\varphi_{k}(\delta)$ is a decreasing function in $\delta$ and satisfies

$$
\lim _{k \rightarrow \infty} \varphi_{k}(\delta)=\left\{\begin{array}{ll}
\infty, & \text { if } \delta<\frac{1}{2} \\
1, & \text { if } \delta=\frac{1}{2} \\
0, & \text { if } \delta>\frac{1}{2}
\end{array} .\right.
$$

Singh [9] (also see Kim and Srivastava [4]) introduced and studied the family of Bazilevič functions $f \in \mathcal{A}$ satisfying the condition:

$$
\operatorname{Re}\left\{\frac{z^{1-\gamma} f^{\prime}(z)}{(f(z))^{1-\gamma}}\right\}>0, \quad(z \in U, \gamma \geq 0) .
$$

According to the Koebe one-quarter theorem (see [3]) "every function $f \in S$ has an inverse $f^{-1}$ which satisfies $f^{-1}(f(z))=z, \quad(z \in U)$ and $f\left(f^{-1}(w)\right)=w$, $\left(|w|<r_{0}(f), r_{0}(f) \geq \frac{1}{4}\right)$, where

$$
g(w)=f^{-1}(w)=w-a_{2} w^{2}+\left(2 a_{2}^{2}-a_{3}\right) w^{3}-\left(5 a_{2}^{3}-5 a_{2} a_{3}+a_{4}\right) w^{4}+\cdots .
$$

For $f \in \mathcal{A}$, if both $f$ and $f^{-1}$ are univalent in $U$, we say that $f$ bi-univalent function in $U$. We indicate by $\Sigma$ the family of bi-univalent functions in $U$ given by (1.1). In fact, Srivastava et al. [18] have actually revived the study of holomorphic and biunivalent functions in recent years. Some examples of functions in the family $\Sigma$ are

$$
\frac{z}{1-z}, \quad \frac{1}{2} \log \left(\frac{1+z}{1-z}\right) \text { and }-\log (1-z)
$$

with the corresponding inverse functions

$$
\frac{w}{1+w}, \quad \frac{e^{2 w}-1}{e^{2 w}+1} \text { and } \frac{e^{w}-1}{e^{w}},
$$

respectively. Other common examples of functions is not a member of $\Sigma$ are

$$
z-\frac{z^{2}}{2} \text { and } \frac{z}{1-z^{2}}
$$


Recently, many authors introduced various subfamilies of the bi-univalent functions family $\Sigma$ and investigated upper bounds for the first two coefficients $\left|a_{2}\right|$ and $\left|a_{3}\right|$ in the Taylor-Maclaurin series expansion (1.1) (see, for example [1,5,10-17,19-24]).

We require the following lemma that will be used to prove our main results.

Lemma 1 ([3]). If $h \in \mathcal{P}$, then $\left|c_{k}\right| \leq 2$ for each $k \in \mathbb{N}$, where $\mathcal{P}$ is the family of all functions h holomorphic in $U$ for which

$$
\operatorname{Re}(h(z))>0, \quad(z \in U),
$$

where

$$
h(z)=1+c_{1} z+c_{2} z^{2}+\cdots, \quad(z \in U) .
$$

\section{CoefFicient ESTIMATES FoR THE FUNCTIONS FAMILY $\Omega_{\Sigma}(\lambda, \gamma, \delta ; \alpha)$}

Definition 1. A function $f \in \Sigma$ given by (1.1) is called in the family $\Omega_{\Sigma}(\lambda, \gamma, \delta ; \alpha)$ if it fulfils the conditions:

$$
\left|\arg \left[\frac{1}{2}\left(\frac{z^{1-\gamma}\left(f * I_{\delta}\right)^{\prime}(z)}{\left(\left(f * I_{\delta}\right)(z)\right)^{1-\gamma}}+\left(\frac{z^{1-\gamma}\left(f * I_{\delta}\right)^{\prime}(z)}{\left(\left(f * I_{\delta}\right)(z)\right)^{1-\gamma}}\right)^{\frac{1}{\lambda}}\right)\right]\right|<\frac{\alpha \pi}{2}, \quad(z \in U)
$$

and

$$
\left|\arg \left[\frac{1}{2}\left(\frac{w^{1-\gamma}\left(g * I_{\delta}\right)^{\prime}(w)}{\left(\left(g * I_{\delta}\right)(w)\right)^{1-\gamma}}+\left(\frac{w^{1-\gamma}\left(g * I_{\delta}\right)^{\prime}(w)}{\left(\left(g * I_{\delta}\right)(w)\right)^{1-\gamma}}\right)^{\frac{1}{\lambda}}\right)\right]\right|<\frac{\alpha \pi}{2}, \quad(w \in U),
$$

$$
(0<\alpha \leq 1,0<\lambda \leq 1, \gamma \geq 0,0 \leq \delta<1),
$$

where the function $g=f^{-1}$ is given by (1.2).

Remark 1. It should be remarked that the family $\Omega_{\Sigma}(\lambda, \gamma, \delta ; \alpha)$ is a generalization of well-known families consider earlier. These families are:

(1) For $\lambda=1$ and $\delta=\frac{1}{2}$, the family $\Omega_{\Sigma}(\lambda, \gamma, \delta ; \alpha)$ reduce to the family $P_{\Sigma}(\alpha, \gamma)$ which was introduced by Prema and Keerthi [7];

(2) For $\lambda=1, \gamma=0$ and $\delta=\frac{1}{2}$, the family $\Omega_{\Sigma}(\lambda, \gamma, \delta ; \alpha)$ reduce to the family $S_{\Sigma}^{*}(\alpha)$ which was given by Brannan and Taha [2];

(3) For $\lambda=\gamma=1$ and $\delta=\frac{1}{2}$, the family $\Omega_{\Sigma}(\lambda, \gamma, \delta ; \alpha)$ reduce to the family $\mathcal{H}_{\Sigma}^{\alpha}$ which was investigated by Srivastava et al. [18].

Theorem 1. Let $f \in \Omega_{\Sigma}(\lambda, \gamma, \delta ; \alpha) \quad(0<\alpha \leq 1,0<\lambda \leq 1, \gamma \geq 0,0 \leq \delta<1)$ be given by (1.1). Then

$$
\left|a_{2}\right| \leq \frac{2 \alpha \lambda}{\sqrt{\alpha \lambda(\gamma+2)(\lambda+1)(1-\delta)(2 \gamma(1-\delta)+1)+\Upsilon(\alpha, \lambda)(\gamma+1)^{2}(1-\delta)^{2}}}
$$


and

$$
\left|a_{3}\right| \leq \frac{4 \alpha \lambda}{(\lambda+1)(1-\delta)}\left[\frac{\alpha \lambda}{(\gamma+1)^{2}(\lambda+1)(1-\delta)}+\frac{1}{(\gamma+2)(3-2 \delta)}\right]
$$

where

$$
\Upsilon(\alpha, \lambda)=2 \alpha(1-\lambda)+(1-\alpha)(\lambda+1)^{2} .
$$

Proof. It follows from conditions (2.1) and (2.2) that

$$
\frac{1}{2}\left(\frac{z^{1-\gamma}\left(f * I_{\delta}\right)^{\prime}(z)}{\left(\left(f * I_{\delta}\right)(z)\right)^{1-\gamma}}+\left(\frac{z^{1-\gamma}\left(f * I_{\delta}\right)^{\prime}(z)}{\left(\left(f * I_{\delta}\right)(z)\right)^{1-\gamma}}\right)^{\frac{1}{\lambda}}\right)=[p(z)]^{\alpha}
$$

and

$$
\frac{1}{2}\left(\frac{w^{1-\gamma}\left(g * I_{\delta}\right)^{\prime}(w)}{\left(\left(g * I_{\delta}\right)(w)\right)^{1-\gamma}}+\left(\frac{w^{1-\gamma}\left(g * I_{\delta}\right)^{\prime}(w)}{\left(\left(g * I_{\delta}\right)(w)\right)^{1-\gamma}}\right)^{\frac{1}{\lambda}}\right)=[q(w)]^{\alpha},
$$

where $g=f^{-1}$ and $p, q$ in $\mathcal{P}$ have the following series representations:

$$
p(z)=1+p_{1} z+p_{2} z^{2}+p_{3} z^{3}+\cdots
$$

and

$$
q(w)=1+q_{1} w+q_{2} w^{2}+q_{3} w^{3}+\cdots .
$$

Comparing the corresponding coefficients of (2.4) and (2.5) yields

$$
\begin{gathered}
\frac{(\gamma+1)(\lambda+1)(1-\delta)}{\lambda} a_{2}=\alpha p_{1} \\
\frac{(\gamma+2)(\lambda+1)(1-\delta)(3-2 \delta)}{2 \lambda} a_{3} \\
+\frac{\left[\lambda(\gamma+2)(\gamma-1)(\lambda+1)+(\gamma+1)^{2}(1-\lambda)\right](1-\delta)^{2}}{\lambda^{2}} a_{2}^{2}=\alpha p_{2}+\frac{\alpha(\alpha-1)}{2} p_{1}^{2} \\
-\frac{(\gamma+1)(\lambda+1)(1-\delta)}{\lambda} a_{2}=\alpha q_{1}
\end{gathered}
$$

and

$$
\begin{aligned}
& \frac{(\gamma+2)(\lambda+1)(1-\delta)(3-2 \delta)}{2 \lambda}\left(2 a_{2}^{2}-a_{3}\right) \\
& +\frac{\left[\lambda(\gamma+2)(\gamma-1)(\lambda+1)+(\gamma+1)^{2}(1-\lambda)\right](1-\delta)^{2}}{\lambda^{2}} a_{2}^{2}=\alpha q_{2}+\frac{\alpha(\alpha-1)}{2} q_{1}^{2} .
\end{aligned}
$$

In view of (2.8) and (2.10), we conclude that

$$
p_{1}=-q_{1}
$$


and

$$
\frac{2(\gamma+1)^{2}(\lambda+1)^{2}(1-\delta)^{2}}{\lambda^{2}} a_{2}^{2}=\alpha^{2}\left(p_{1}^{2}+q_{1}^{2}\right) .
$$

Also, by using (2.9) and (2.11), together with (2.13), we find that

$$
\begin{aligned}
& \left(\frac{(\gamma+2)(\lambda+1)(1-\delta)(3-2 \delta)}{\lambda}+\frac{2\left[\lambda(\gamma+2)(\gamma-1)(\lambda+1)+(\gamma+1)^{2}(1-\lambda)\right](1-\delta)^{2}}{\lambda^{2}}\right) a_{2}^{2} \\
& =\alpha\left(p_{2}+q_{2}\right)+\frac{\alpha(\alpha-1)}{2}\left(p_{1}^{2}+q_{1}^{2}\right)=\alpha\left(p_{2}+q_{2}\right)+\frac{(\alpha-1)(\gamma+1)^{2}(\lambda+1)^{2}(1-\delta)^{2}}{\alpha \lambda^{2}} a_{2}^{2} .
\end{aligned}
$$

Further computations show that

$$
a_{2}^{2}=\frac{\alpha^{2} \lambda^{2}\left(p_{2}+q_{2}\right)}{\alpha \lambda(\gamma+2)(\lambda+1)(1-\delta)(2 \gamma(1-\delta)+1)+\Upsilon(\alpha, \lambda)(\gamma+1)^{2}(1-\delta)^{2}},
$$

where $\Upsilon(\alpha, \lambda)$ is given by (2.3).

By taking the absolute value of (2.14) and applying Lemma 1 for the coefficients $p_{2}$ and $q_{2}$, we have

$$
\left|a_{2}\right| \leq \frac{2 \alpha \lambda}{\sqrt{\alpha \lambda(\gamma+2)(\lambda+1)(1-\delta)(2 \gamma(1-\delta)+1)+\Upsilon(\alpha, \lambda)(\gamma+1)^{2}(1-\delta)^{2}}} .
$$

To determinate the bound on $\left|a_{3}\right|$, by subtracting (2.11) from (2.9), we get

$$
\frac{(\gamma+2)(\lambda+1)(1-\delta)(3-2 \delta)}{\lambda}\left(a_{3}-a_{2}^{2}\right)=\alpha\left(p_{2}-q_{2}\right)+\frac{\alpha(\alpha-1)}{2}\left(p_{1}^{2}-q_{1}^{2}\right) \text {. }
$$

Now, substituting the value of $a_{2}^{2}$ from (2.13) into (2.15) and using (2.12), we deduce that

$$
a_{3}=\frac{\alpha^{2} \lambda^{2}\left(p_{1}^{2}+q_{1}^{2}\right)}{2(\gamma+1)^{2}(\lambda+1)^{2}(1-\delta)^{2}}+\frac{\alpha \lambda\left(p_{2}-q_{2}\right)}{(\gamma+2)(\lambda+1)(1-\delta)(3-2 \delta)} .
$$

Taking the absolute value of (2.16) and applying Lemma 1 once again for the coefficients $p_{1}, p_{2}, q_{1}$ and $q_{2}$, it follows that

$$
\left|a_{3}\right| \leq \frac{4 \alpha \lambda}{(\lambda+1)(1-\delta)}\left[\frac{\alpha \lambda}{(\gamma+1)^{2}(\lambda+1)(1-\delta)}+\frac{1}{(\gamma+2)(3-2 \delta)}\right]
$$

Remark 2. In Theorem 1, if we choose

1) $\lambda=1$ and $\delta=\frac{1}{2}$, then we have the results which was given by Prema and Keerthi [7, Theorem 2.2];

(2) $\lambda=1, \gamma=0$ and $\delta=\frac{1}{2}$, then we have the results obtained by Murugusundaramoorthy et al. [6, Corollary 6]; 
(3) $\lambda=\gamma=1$ and $\delta=\frac{1}{2}$, then we obtain the results obtained by Srivastava et al. [18, Theorem 1].

\section{Coefficient estimates For THE FUnCtions FAMily $\Omega_{\Sigma}^{*}(\lambda, \gamma, \delta ; \beta)$}

Definition 2. A function $f \in \Sigma$ given by (1.1) is called in the family $\Omega_{\Sigma}^{*}(\lambda, \gamma, \delta ; \beta)$ if it fulfills the conditions:

$$
\operatorname{Re}\left\{\frac{1}{2}\left(\frac{z^{1-\gamma}\left(f * I_{\delta}\right)^{\prime}(z)}{\left(\left(f * I_{\delta}\right)(z)\right)^{1-\gamma}}+\left(\frac{z^{1-\gamma}\left(f * I_{\delta}\right)^{\prime}(z)}{\left(\left(f * I_{\delta}\right)(z)\right)^{1-\gamma}}\right)^{\frac{1}{\lambda}}\right)\right\}>\beta, \quad(z \in U)
$$

and

$$
\operatorname{Re}\left\{\frac{1}{2}\left(\frac{w^{1-\gamma}\left(g * I_{\delta}\right)^{\prime}(w)}{\left(\left(g * I_{\delta}\right)(w)\right)^{1-\gamma}}+\left(\frac{w^{1-\gamma}\left(g * I_{\delta}\right)^{\prime}(w)}{\left(\left(g * I_{\delta}\right)(w)\right)^{1-\gamma}}\right)^{\frac{1}{\lambda}}\right)\right\}>\beta, \quad(w \in U),
$$

$$
(0 \leq \beta<1,0<\lambda \leq 1, \gamma \geq 0,0 \leq \delta<1),
$$

where the function $g=f^{-1}$ is given by (1.2).

Remark 3. It should be remarked that the family $\Omega_{\Sigma}^{*}(\lambda, \gamma, \delta ; \beta)$ is a generalization of well-known families consider earlier. These families are:

(1) For $\lambda=1$ and $\delta=\frac{1}{2}$, the family $\Omega_{\Sigma}^{*}(\lambda, \gamma, \delta ; \beta)$ reduce to the family $P_{\Sigma}(\beta, \gamma)$ which was introduced by Prema and Keerthi [7];

(2) For $\lambda=1, \gamma=0$ and $\delta=\frac{1}{2}$, the family $\Omega_{\Sigma}^{*}(\lambda, \gamma, \delta ; \beta)$ reduce to the family $S_{\Sigma}^{*}(\beta)$ which was given by Brannan and Taha [2];

(3) For $\lambda=\gamma=1$ and $\delta=\frac{1}{2}$, the family $\Omega_{\Sigma}^{*}(\lambda, \gamma, \delta ; \beta)$ reduce to the family $\mathcal{H}_{\Sigma}(\beta)$ which was investigated by Srivastava et al. [18].

Theorem 2. Let $f \in \Omega_{\Sigma}^{*}(\lambda, \gamma, \delta ; \beta)(0 \leq \beta<1,0<\lambda \leq 1, \gamma \geq 0,0 \leq \delta<1)$ be given by (1.1). Then

$$
\left|a_{2}\right| \leq \frac{2 \lambda \sqrt{1-\beta}}{\sqrt{\lambda(\gamma+2)(\lambda+1)(1-\delta)(2 \gamma(1-\delta)+1)+2(1-\lambda)(\gamma+1)^{2}(1-\delta)^{2}}}
$$

and

$$
\left|a_{3}\right| \leq \frac{4 \lambda(1-\beta)}{(\lambda+1)(1-\delta)}\left[\frac{\lambda(1-\beta)}{(\gamma+1)^{2}(\lambda+1)(1-\delta)}+\frac{1}{(\gamma+2)(3-2 \delta)}\right] .
$$

Proof. In the light of the conditions (3.1) and (3.2), there are $p, q \in \mathcal{P}$ such that

$$
\frac{1}{2}\left(\frac{z^{1-\gamma}\left(f * I_{\delta}\right)^{\prime}(z)}{\left(\left(f * I_{\delta}\right)(z)\right)^{1-\gamma}}+\left(\frac{z^{1-\gamma}\left(f * I_{\delta}\right)^{\prime}(z)}{\left(\left(f * I_{\delta}\right)(z)\right)^{1-\gamma}}\right)^{\frac{1}{\lambda}}\right)=\beta+(1-\beta) p(z)
$$


and

$$
\frac{1}{2}\left(\frac{w^{1-\gamma}\left(g * I_{\delta}\right)^{\prime}(w)}{\left(\left(g * I_{\delta}\right)(w)\right)^{1-\gamma}}+\left(\frac{w^{1-\gamma}\left(g * I_{\delta}\right)^{\prime}(w)}{\left(\left(g * I_{\delta}\right)(w)\right)^{1-\gamma}}\right)^{\frac{1}{\lambda}}\right)=\beta+(1-\beta) q(w),
$$

where $p(z)$ and $q(w)$ have the forms (2.6) and (2.7), respectively. Comparing the corresponding coefficients in (3.3) and (3.4) yields

$$
\begin{gathered}
\frac{(\gamma+1)(\lambda+1)(1-\delta)}{\lambda} a_{2}=(1-\beta) p_{1}, \\
\frac{(\gamma+2)(\lambda+1)(1-\delta)(3-2 \delta)}{2 \lambda} a_{3} \\
+\frac{\left[\lambda(\gamma+2)(\gamma-1)(\lambda+1)+(\gamma+1)^{2}(1-\lambda)\right](1-\delta)^{2}}{\lambda^{2}} a_{2}^{2}=(1-\beta) p_{2}, \\
-\frac{(\gamma+1)(\lambda+1)(1-\delta)}{\lambda} a_{2}=(1-\beta) q_{1}
\end{gathered}
$$

and

$$
\begin{aligned}
& \frac{(\gamma+2)(\lambda+1)(1-\delta)(3-2 \delta)}{2 \lambda}\left(2 a_{2}^{2}-a_{3}\right) \\
& +\frac{\left[\lambda(\gamma+2)(\gamma-1)(\lambda+1)+(\gamma+1)^{2}(1-\lambda)\right](1-\delta)^{2}}{\lambda^{2}} a_{2}^{2}=(1-\beta) q_{2} .
\end{aligned}
$$

From (3.5) and (3.7), we get

$$
p_{1}=-q_{1}
$$

and

$$
\frac{2(\gamma+1)^{2}(\lambda+1)^{2}(1-\delta)^{2}}{\lambda^{2}} a_{2}^{2}=(1-\beta)^{2}\left(p_{1}^{2}+q_{1}^{2}\right) .
$$

Adding (3.6) and (3.8), we obtain

$$
\begin{aligned}
& \left(\frac{(\gamma+2)(\lambda+1)(1-\delta)(3-2 \delta)}{\lambda}\right. \\
& \left.+\frac{2\left[\lambda(\gamma+2)(\gamma-1)(\lambda+1)+(\gamma+1)^{2}(1-\lambda)\right](1-\delta)^{2}}{\lambda^{2}}\right) a_{2}^{2}=(1-\beta)\left(p_{2}+q_{2}\right) .
\end{aligned}
$$

Hence, we find that

$$
a_{2}^{2}=\frac{\lambda^{2}(1-\beta)\left(p_{2}+q_{2}\right)}{\lambda(\gamma+2)(\lambda+1)(1-\delta)(2 \gamma(1-\delta)+1)+2(1-\lambda)(\gamma+1)^{2}(1-\delta)^{2}} .
$$


By applying Lemma 1 for the coefficients $p_{2}$ and $q_{2}$, we deduce that

$$
\left|a_{2}\right| \leq \frac{2 \lambda \sqrt{1-\beta}}{\sqrt{\lambda(\gamma+2)(\lambda+1)(1-\delta)(2 \gamma(1-\delta)+1)+2(1-\lambda)(\gamma+1)^{2}(1-\delta)^{2}}} .
$$

To determinate the bound on $\left|a_{3}\right|$, by subtracting (3.8) from (3.6), we get

$$
\frac{(\gamma+2)(\lambda+1)(1-\delta)(3-2 \delta)}{\lambda}\left(a_{3}-a_{2}^{2}\right)=(1-\beta)\left(p_{2}-q_{2}\right),
$$

or equivalently

$$
a_{3}=a_{2}^{2}+\frac{\lambda(1-\beta)\left(p_{2}-q_{2}\right)}{(\gamma+2)(\lambda+1)(1-\delta)(3-2 \delta)} .
$$

Substituting the value of $a_{2}^{2}$ from (3.10) into (3.12), it follows that

$$
a_{3}=\frac{\lambda^{2}(1-\beta)^{2}\left(p_{1}^{2}+q_{1}^{2}\right)}{2(\gamma+1)^{2}(\lambda+1)^{2}(1-\delta)^{2}}+\frac{\lambda(1-\beta)\left(p_{2}-q_{2}\right)}{(\gamma+2)(\lambda+1)(1-\delta)(3-2 \delta)} .
$$

By applying Lemma 1 once again for the coefficients $p_{1}, p_{2}, q_{1}$ and $q_{2}$, we deduce that

$$
\left|a_{3}\right| \leq \frac{4 \lambda(1-\beta)}{(\lambda+1)(1-\delta)}\left[\frac{\lambda(1-\beta)}{(\gamma+1)^{2}(\lambda+1)(1-\delta)}+\frac{1}{(\gamma+2)(3-2 \delta)}\right] .
$$

Remark 4. In Theorem 2, if we choose

(1) $\lambda=1$ and $\delta=\frac{1}{2}$, then we have the results which was given by Prema and Keerthi [7, Theorem 3.2];

(2) $\lambda=1, \gamma=0$ and $\delta=\frac{1}{2}$, then we have the results obtained by Murugusundaramoorthy et al. [6, Corollary 7];

(3) $\lambda=\gamma=1$ and $\delta=\frac{1}{2}$, then we obtain the results obtained by Srivastava et al. [18, Theorem 2].

\section{ACKNOWLEDGEMENT}

The author would like to thank the referee(s) for their helpful comments and suggestions.

\section{REFERENCES}

[1] E. A. Adegani, S. Bulut, and A. A. Zireh, "Coefficient estimates for a subclass of analytic bi-univalent functions," Bull. Korean Math. Soc., vol. 55, pp. 405-413, 2018, doi: 10.4134/BKMS.b170051.

[2] D. A. Brannan and T. S. Taha, "On some classes of bi-univalent functions," Studia Univ. BabesBolyai Math., vol. 31, pp. 70-77, 1986.

[3] P. L. Duren, Univalent Functions. New York, Berlin, Heidelberg and Tokyo: Springer Verlag, 1983. 
[4] Y. C. Kim and H. M. Srivastava, "The Hardy space of a certain subclass of Bazilevič functions," Appl. Math. Comput., vol. 183, pp. 1201-1207, 2006, doi: 10.1016/j.amc.2006.06.044.

[5] N. Magesh and S. Bulut, "Chebyshev polynomial coefficient estimates for a class of analytic biunivalent functions related to pseudo-starlike functions," Afr. Mat., vol. 29, pp. 203-209, 2018, doi: 10.1007/s13370-017-0535-3.

[6] G. Murugusundaramoorthy, N. Magesh, and V. Prameela, "Coefficient bounds for certain subclasses of bi-univalent function," Abstr. Appl. Anal., vol. 2013, pp. 1-3, 2013, doi: 10.1155/2013/573017.

[7] S. Prema and B. S. Keerthi, "Coefficient bounds for certain subclasses of analytic function," $J$. Math. Anal., vol. 4, pp. 22-27, 2013.

[8] S. Ruscheweyh, "Linear operators between classes of prestarlike functions," Comment. Math. Helv., vol. 52, pp. 497-509, 1977, doi: 10.1007/BF02567382.

[9] R. Singh, "On Bazilevič functions," Proc. Amer. Math. Soc., vol. 38, pp. 261-271, 1973.

[10] H. M. Srivastava, "Operators of basic (or q-) calculus and fractional q-calculus and their applications in geometric function theory of complex analysis," Iran. J. Sci. Technol. Trans. A: Sci., vol. 44, pp. 327-344, 2020, doi: 10.1007/s40995-019-00815-0.

[11] H. M. Srivastava and D. Bansal, "Coefficient estimates for a subclass of analytic and bi-univalent functions,” J. Egyptian Math. Soc., vol. 23, pp. 242-246, 2015, doi: 10.1016/j.joems.2014.04.002.

[12] H. M. Srivastava, S. Bulut, M. Caglar, and N. Yagmur, "Coefficient estimates for a general subclass of analytic and bi-univalent functions," Filomat, vol. 27, pp. 831-842, 2013, doi: 10.2298/FIL1305831S.

[13] H. M. Srivastava, Ş. Altınkaya, and S. Yalçin, "Certain subclasses of bi-univalent functions associated with the Horadam polynomials," Iran. J. Sci. Technol. Trans. A Sci., vol. 43, pp. 1873-1879, 2019, doi: 10.1007/s40995-018-0647-0.

[14] H. M. Srivastava, S. S. Eker, and R. M. Ali, "Coefficient bounds for a certain class of analytic and bi-univalent functions," Filomat, vol. 29, pp. 1839-1845, 2015, doi: 10.2298/FIL1508839S.

[15] H. M. Srivastava, S. S. Eker, S. G. Hamidi, and J. M. Jahangiri, "Faber polynomial coefficient estimates for bi-univalent functions defined by the Tremblay fractional derivative operator," Bull. Iranian Math. Soc., vol. 44, pp. 149-157, 2018, doi: 10.1007/s41980-018-0011-3.

[16] H. M. Srivastava, S. Gaboury, and F. Ghanim, "Coefficient estimates for some general subclasses of analytic and bi-univalent functions," Africa Mat., vol. 28, pp. 693-706, 2017, doi: 10.1007/s13370-016-0478-0.

[17] H. M. Srivastava, S. Gaboury, and F. Ghanim, "Coefficient estimates for a general subclass of analytic and bi-univalent functions of the Ma-Minda type," Rev. Real Acad. Cienc. Exactas Fís. Natur. Ser. A Mat. (RACSAM), vol. 112, pp. 1157-1168, 2018, doi: 10.1007/S13398-017-0416-5.

[18] H. M. Srivastava, A. K. Mishra, and P. Gochhayat, "Certain subclasses of analytic and bi-univalent functions,” Appl. Math. Lett., vol. 23, pp. 1188-1192, 2010, doi: 10.1016/j.aml.2010.05.009.

[19] H. M. Srivastava, A. Motamednezhad, and E. A. Adegan, "Faber polynomial coefficient estimates for bi-univalent functions defined by using differential subordination and a certain fractional derivative operator," Mathematics, vol. 8, pp. 1-12, 2020, doi: 10.3390/math8020172.

[20] H. M. Srivastava, G. Murugusundaramoorthy, and K. Vijaya, "Coefficient estimates for some families of bi-Bazilevič functions of the Ma-Minda type involving the Hohlov operator," J. Class. Anal., vol. 2, pp. 167-181, 2013, doi: 10.7153/jca-02-14.

[21] H. M. Srivastava, F. M. Sakar, and H. O. Güney, "Some general coefficient estimates for a new class of analytic and bi-univalent functions defined by a linear combination," Filomat, vol. 32, pp. 1313-1322, 2018, doi: 10.2298/FIL1804313S.

[22] H. M. Srivastava and A. K. Wanas, "Initial Maclaurin coefficient bounds for new subclasses of analytic and m-fold symmetric bi-univalent functions defined by a linear combination," Kyungpook Math. J., vol. 59, pp. 493-503, 2019, doi: 10.5666/KMJ.2019.59.3.493. 
[23] A. K. Wanas and A. L. Alina, "Applications of Horadam polynomials on Bazilevič bi-univalent function satisfying subordinate conditions," Journal of Physics: Conf. Series, vol. 1294, pp. 1-6, 2019, doi: 10.1088/1742-6596/1294/3/032003.

[24] P. Zaprawa, "On the Fekete-Szegố problem for classes of bi-univalent functions," Bull. Belg. Math. Soc. Simon Stevin, vol. 21, pp. 169-178, 2014, doi: 10.36045/bbms/1394544302.

Author's address

Abbas Kareem Wanas

Department of Mathematics, College of Science, University of Al-Qadisiyah, Iraq

E-mail address: abbas.kareem.wequ.edu.iq 